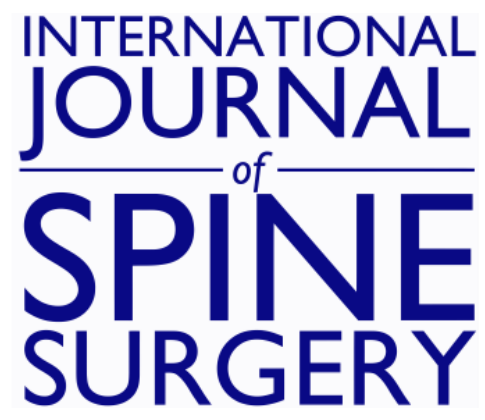

\title{
Prevalence of Venous Thromboembolism Following Acute Spinal Cord Injury in an Urban Inner City Hospital
}

Andrew Lowery, Akul Patel, Robert Ames, Frederick Ramsey, Bridget Slattery and Theresa Pazionis

Int J Spine Surg 2021, 15 (3) 562-569

doi: https://doi.org/10.14444/8076

http://ijssurgery.com/content/15/3/562

This information is current as of April 26, 2023.

Email Alerts Receive free email-alerts when new articles cite this article. Sign up at: http://ijssurgery.com/alerts 


\title{
Prevalence of Venous Thromboembolism Following Acute Spinal Cord Injury in an Urban Inner City Hospital
}

\author{
ANDREW LOWERY, MD, ${ }^{1}$ AKUL PATEL, MD,${ }^{2}$ ROBERT AMES, MD,${ }^{2}$ FREDERICK RAMSEY, PHD,${ }^{3}$ \\ BRIDGET SLATTERY, BS, MHS, ${ }^{2}$ THERESA PAZIONIS, MD ${ }^{2}$ \\ ${ }^{I}$ Temple University School of Medicine, Philadelphia, Pennsylvania ${ }^{2}$ Temple University Department of Orthopedic Surgery and Sports Medicine, Philadelphia, \\ Pennsylvania ${ }^{3}$ Department of Clinical Sciences, Temple University School of Medicine, Philadelphia, Pennsylvania
}

\begin{abstract}
Background: Venous thromboembolism (VTE) is known to occur at high rates in patients who suffer spinal cord injury (SCI). Large population studies in the United States have shown a prevalence of approximately $4-5 \%$, with some studies suggesting higher prevalence. While the specific mechanism behind increased VTE incidence in the SCI population is unknown, it is likely tied to immobility and functional decline. Previous studies have also linked social determinants of health to higher VTE prevalence in certain populations. The purpose of this study is to determine the prevalence of VTE after acute SCI in the inner urban city population and to identify factors that place patients at increased VTE risk.

Methods: Patients who suffered SCI between 2014 and 2019 were identified from one inner city urban hospital. A retrospective chart review was completed to record the development of VTE after SCI. Medical comorbidities, type of thromboprophylaxis used, patient demographics, injury characteristics, and postinjury sequelae were compared between individuals who did and did not develop VTE.

Results: A total of 148 patients were included. These patients were from a low socioeconomic demographic when compared with the larger US population. Average household income based on zip code data for included patients was \$56 647, \$30 315 below the national average. The prevalence of VTE in this patient population was $19.59 \%$. Weight, deep vein thrombosis history, chemoprophylaxis use, mobility impairment postinjury, neurologic level of SCI, malignancy history, and history of smokeless tobacco were associated with VTE (all $P<.05$ ). Low molecular weight heparin (LMWH) use was associated with fewer occurrences of VTE $(P<.001)$.

Conclusion: Patients from our urban inner city hospital have a higher VTE prevalence after SCI than shown in previous US-based studies. These patients may be at increased risk due to increased numbers of medical comorbidities, social factors, or undiagnosed medical conditions. Thromboprophylaxis with LMWH appears to lower the risk of VTE after SCI.

Level of Evidence: 3.

Clinical Relevance: Patients with the previously mentioned risk factors are at increased risk for VTE development during their acute recovery process. These patients should have a much lower screening threshold for VTE evaluation and likely would benefit from more routine screening to prevent complications related to VTE development or progression. Furthermore, these patients should, when medically appropriate, be treated with low molecular weight heparin for VTE prophylaxis as previously recommended by the Consortium for Spinal Cord Medicine.
\end{abstract}

\section{Complications}

Keywords: venous thromboembolism, acute spinal cord injury, thromboprophylaxis, urban inner city, blunt spinal trauma, penetrating spinal trauma, cervical spine, thoracic spine, lumbar spine

\section{INTRODUCTION}

Patients who suffer acute spinal cord injury (SCI) are at high risk of complications such as venous thromboembolism (VTE), which significantly increases their morbidity and mortality. ${ }^{1-3}$ VTE development is thought to relate to Virchow's triad of hypercoagulability, disrupted endothelium, and turbulent blood flow. Prophylaxis for the prevention of VTE focuses on decreasing hypercoagulability through chemical or mechanical means. A study by Aito et $\mathrm{al}^{4}$ found that administration of low molecular weight heparin (LMWH) within 72 hours of injury reduced VTE incidence. Despite routine prophylaxis, patients who suffer acute SCI have a higher prevalence of VTE than patients who suffer other traumatic injuries with sparing of the spinal cord. ${ }^{1}$ Although VTE is a known complication of acute SCI, the exact prevalence and factors that contribute to it remain under investigation. The rate 
of VTE following acute SCI in recent studies has ranged from $4.3 \%$ to $23.4 \%$ internationally. In the United States, recent studies suggest a VTE rate of approximately $5 \% .^{5-8}$

The differences reported in VTE prevalence could be due to differences in baseline patient demographics. Jones et $\mathrm{al}^{5}$ found that male sex, complete tetraplegia, obesity, and age greater than 70 were associated with a higher likelihood of developing a VTE. They also mention a correlation between race and VTE development and the possibility that social factors or underused medical care may influence VTE development. This was supported by their finding that Medicaid insurance was also associated with increased VTE risk. ${ }^{5}$

VTE prevention in patients with acute SCI is challenging. Multiple studies have suggested that LMWH is the most effective method of chemoprophylaxis and that mechanical prophylaxis alone is not sufficient. ${ }^{9-12}$ This is consistent with the recommendations provided by the Consortium for Spinal Cord Medicine for VTE prevention after SCI. ${ }^{9}$ Teasell et al $^{13}$ suggested in their systematic review that VTE chemoprophylaxis with LMWH should be considered first line with an adjusted dose of unfractionated heparin useful as an alternative if rapid reversal is needed due to increased bleeding risk. This is also supported by Christie et $\mathrm{al}^{14}$ who suggested that LMWH should be started within 72 hours of SCI in their systematic review to assess the importance of timing of thromboprophylaxis. While these data and set of guidelines are useful, they do not take into account patient risk factors or medical comorbidities that can increase the risk of VTE after SCI. While most patients likely respond similarly to thromboprophylaxis, little information is available about the efficacy of traditional thromboprophylaxis in patients at a higher baseline risk.

Here, we assess the prevalence of VTE in acute SCI patients in one urban inner city hospital. We predict that the prevalence of VTE in this population is greater than that found in prior literature owing to the inherent medical comorbidities and risk factors present in this demographic. The secondary purpose of this study is to assess specific medical and social risk factors for VTE development and to assess the effectiveness of various forms of chemoprophylaxis in this patient sample.

\section{METHODS}

A retrospective chart review was completed to identify patients between the ages of 19 and 89 presenting to one urban inner city hospital between January 2014 and October 2019 with an acute SCI. Institutional Review Board approval was received November 2019. ICD10 codes used to complete the search were S14, S24, and S34, which correspond to cervical, thoracic, and lumbar SCI, respectively. Patients with a lack of clinical or radiographic characteristics suggesting acute SCI were excluded. In addition, those who expired within 2 weeks of hospital admission and could not be assessed for VTE development, who had a previous SCI, and who left the hospital against medical advice and thus had no records to assess for development of VTE were also excluded from analysis. Types of VTE included deep vein thrombosis (DVT) of the upper and lower extremity and any form of pulmonary embolus (PE). Positive VTE was assessed using any available imaging reports or report by clinicians in the electronic medical record if an imaging report was not available.

Patients $(n=148)$ were divided into two groups consisting of those who developed VTE $(n=29)$ and those who did not $(n=119)$. Recorded patient demographics included age, race, sex, body mass index (BMI), weight, height, and ethnicity. Other data collected on each patient included medical comorbidities, mechanism of injury with differentiation between blunt and penetrating injuries, level of spine injury, time from SCI to VTE, previous history of DVTs, the form of DVT chemoprophylaxis used, including the use of sequential compression devices (SCDs), and surgical history within the previous 3 months of injury. Smoking history, including the use of smokeless nicotine and illicit substance use, was also recorded. Other social factors assessed included employment status and the use of government-assisted healthcare coverage. The presence of thrombocytosis was recorded on the day of admission and, if applicable, the day before VTE diagnosis. Time to VTE was assessed by measuring the number of days from injury, which was typically the day of admission, to the day of diagnosis. The presence and degree of mobility impairment was also recorded.

Finally, Socioeconomic demographics were estimated for the entire patient cohort. Average patient household income was inferred using residential zip codes and data from the US Census Bureau. ${ }^{15}$ 
Table 1. Baseline patient demographics by venous thromboembolism development after spinal cord injury.

\begin{tabular}{|c|c|c|c|c|}
\hline & VTE & No VTE & All Patients & $P$ Value \\
\hline Age, average $\pm \mathrm{SD}, \mathrm{y}$ & $45.1 \pm 18.3$ & $45.7 \pm 18.0$ & $44.6 \pm 17.9$ & .883 \\
\hline Height, average $\pm \mathrm{SD}, \mathrm{m}$ & $1.7 \pm 0.1$ & $1.7 \pm 0.1$ & $1.7 \pm 0.1$ & .664 \\
\hline Weight, average $\pm \mathrm{SD}, \mathrm{kg}$ & $83.3 \pm 19.7$ & $75.8 \pm 17.5$ & $77.2 \pm 18.1$ & .048 \\
\hline $\mathrm{BMI}$, average $\pm \mathrm{SD}, \mathrm{kg} / \mathrm{m}^{2}$ & $27.4 \pm 6.6$ & $25.1 \pm 5.5$ & $25.6 \pm 5.7$ & .055 \\
\hline \multicolumn{5}{|l|}{ Sex, $n(\%)$} \\
\hline Male & $26(89.7)$ & $99(83.2)$ & $125(84.5)$ & .764 \\
\hline Female & $3(10.3)$ & $20(16.8)$ & $23(15.5)$ & \\
\hline \multicolumn{5}{|l|}{ Race, $n(\%)$} \\
\hline Caucasian & $5(17.2)$ & $24(20.3)$ & 29 (19.6) & .579 \\
\hline African American & $19(65.5)$ & $69(58.5)$ & $88(59.5)$ & \\
\hline Hispanic & $5(17.2)$ & $14(11.9)$ & $19(12.8)$ & \\
\hline Asian/Pacific Islander & $0(0)$ & $1(0.8)$ & $1(0.7)$ & \\
\hline Other & $0(0)$ & $3(2.5)$ & $3(2)$ & \\
\hline Unknown & $0(0)$ & $8(6.8)$ & $8(5.4)$ & \\
\hline \multicolumn{5}{|l|}{ Ethnicity, $n(\%)$} \\
\hline Hispanic & $5(17.9)$ & $17(15.3)$ & $22(15.8)$ & .791 \\
\hline Non-Hispanic & $22(78.6)$ & $86(77.5)$ & $108(92.3)$ & \\
\hline Unknown & $1(3.6)$ & $8(7.2)$ & $9(7.7)$ & \\
\hline
\end{tabular}

Abbreviations: BMI, body mass index; VTE, venous thromboembolism.

\section{Statistical Methods}

Statistical methods in this study included continuous outcome variables, selected categorical variables, and pairwise analyses of categorical variables. All analyses were performed by a trained statistician. All statistical interpretation of significance was based on the $P$ value of .05 , with less than .05 being significant. All patients $(n=148)$ were included for each variable unless the patient was missing data for that specific variable. Continuous outcome variables included age, height, weight, and BMI with comparison between those who did and those who did not develop VTE after SCI. These were compared using the two-sample $t$-test.

Categorical variables included sex, race, ethnicity, mechanism of injury, spine injury level, DVT history, DVT prophylactic method, presence of mobility impairment after injury, degree of mobility impairment after injury compared with baseline, chronic kidney disease, chronic obstructive pulmonary disease, congestive heart failure, depression, anxiety, surgery within the previous 3 months, cancer history, smoking status, smokeless tobacco use, cocaine use, diabetes, hypertension, myocardial infarction or coronary artery disease, peripheral vascular disease, cerebrovascular attack, and thrombocytosis. These were compared between those who did and did not develop VTE after SCI using the chi-square test or Fisher's exact test when appropriate.

Pairwise analyses of categorical variables was performed for DVT prophylactic methods to compare specific methods within this category.
Spine level of injury also underwent pairwise analysis to compare cervical with thoracic injury. Each pairwise analysis was compared between those who did and did not develop VTE after SCI via the chi-square test or Fisher's exact test when appropriate.

\section{RESULTS}

We identified 208 patients who presented with SCI between January 2014 and October 2019. Of these 208 patients, 148 met the inclusion criteria and were included in the analysis. The remaining 60 patients were excluded for the aforementioned reasons. Patients with missing data were excluded from that specific variable's analysis only. One patient experienced VTE following acute SCI that occurred before our electronic medical record (EMR) use and therefore was excluded from the correlated analyses. One patient lacked data on the type of chemoprophylaxis used and was therefore excluded from this analysis. Two patients had no recorded weight and were therefore excluded from BMI and weight analyses. Several patients had no ethnicity data available and were excluded from those respective analyses.

The overall prevalence of VTE in this study was $19.59 \%$. Average time from causative incident of SCI to VTE was 34.4 days with a standard deviation of 41.7 days. Three patients had VTE occurrence in the range of 100-160 days. The remainder occurred before day 100. Table 1 summarizes patient demographics. The average age of the entire cohort was 44.6 years with a standard deviation of 17.9. 
Table 2. Patient comorbidities and social factors by venous thromboembolism development after spinal cord injury, $n(\%)$.

\begin{tabular}{|c|c|c|c|c|}
\hline & VTE & No VTE & All Patients & $P$ Value \\
\hline \multicolumn{5}{|l|}{ Medical comorbidity } \\
\hline Prior or current malignancy & $6(21.4)$ & $9(7.6)$ & $15(10.2)$ & .04 \\
\hline Type 2 diabetes & $9(31)$ & $27(22.7)$ & $36(24.3)$ & .348 \\
\hline Hypertension & $10(34.5)$ & $41(34.5)$ & $51(34.5)$ & .998 \\
\hline Chronic kidney disease & $6(21.4)$ & $10(8.4)$ & $16(10.9)$ & .083 \\
\hline Chronic obstructive pulmonary disease & $4(14.3)$ & $20(16.8)$ & $24(16.3)$ & .999 \\
\hline Congestive heart failure & $3(10.7)$ & $9(7.6)$ & $12(8.2)$ & .7 \\
\hline Myocardial infarction or coronary artery disease & $7(26.9)$ & $17(17.9)$ & $24(19.8)$ & .168 \\
\hline Peripheral vascular disease & $0(0)$ & $3(2.5)$ & $3(2)$ & .999 \\
\hline Prior stroke & $2(7)$ & $5(4.2)$ & $7(4.8)$ & 618 \\
\hline Presence of thrombocytosis & $4(14.3)$ & $6(5)$ & $10(6.8)$ & .097 \\
\hline History of depression & $5(17.9)$ & $23(19.3)$ & $28(19.0)$ & .844 \\
\hline History of anxiety & $3(10.7)$ & $19(16)$ & $22(15)$ & .844 \\
\hline History of DVT & $5(17.2)$ & $2(1.7)$ & $7(4.7)$ & .003 \\
\hline \multicolumn{5}{|l|}{ Social factors } \\
\hline Smoker & $13(46.4)$ & $60(50.4)$ & $73(49.7)$ & .7 \\
\hline Smokeless tobacco use & $6(21.4)$ & $8(6.7)$ & $14(9.5)$ & .028 \\
\hline Substance abuse & $6(21.4)$ & $24(20.2)$ & $30(20.4)$ & .882 \\
\hline Unemployed & $11(39.3)$ & $51(42.9)$ & $62(41.9)$ & .84 \\
\hline Government-assisted healthcare coverage & $26(92.3)$ & $94(79)$ & $120(81.1)$ & .266 \\
\hline
\end{tabular}

Abbreviations: DVT, deep vein thrombosis; VTE, venous thromboembolism.

Patient weight was significantly associated with VTE development. Individuals with higher weight at the time of their SCI were more likely to develop VTE. The average weight of those who developed VTE was $83.3 \mathrm{~kg}$ with a standard deviation of 19.7 $\mathrm{kg}$ compared with an average of $75.8 \mathrm{~kg}$ with a standard deviation of $17.5 \mathrm{~kg}$ in those who did not $(P<.05)$. BMI trended towards significance. The average BMI of those who developed VTE was 27.4 $\mathrm{kg} / \mathrm{m}^{2}$ versus $25.1 \mathrm{~kg} / \mathrm{m}^{2}$ in those who did not $(P=$ .055). Other patient demographics did not show an association with thromboembolic disease.

Table 2 shows medical comorbidities and patient social factors. Review of medical comorbidities showed that prior or current malignancy was associated with VTE, and $21.4 \%$ of individuals with prior or current malignancy developed VTE versus $7.6 \%$ of those without a history of malignancy $(P<.05)$. The only other medical comorbidity associated with VTE development was a history of DVT. Individuals with prior DVT had a $17.2 \%$ chance of developing VTE compared with a $1.7 \%$ chance in those without DVT history $(P<.05)$. Variables assessed for but without statistical analysis include heparin-induced thrombocytopenia type 2, Factor 5 Leiden, antiphospholipid syndrome, paroxysmal nocturnal hemoglobinuria, protein $\mathrm{C}$ deficiency, and protein S deficiency. No patients in this study were diagnosed with these conditions. Review of patient social factors showed that $21.4 \%$ of patients who developed VTE were using smokeless tobacco compared with $6.7 \%$ of patients who were not $(P<.05)$. Eighty-six percent of patients were using government-assisted healthcare coverage, and $47 \%$ of patients before injury reported being unemployed. There was no statistically significant difference for these two variables among those who did and did not develop a VTE.

Table 3 summarizes injury characteristics; $62.2 \%$ of patients experienced blunt trauma, while the

Table 3. Injury characteristics of patients who did and did not develop venous thromboembolism following spinal cord injury, ( $n$ (\%)).

\begin{tabular}{|c|c|c|c|c|}
\hline Injury Characteristic & VTE & No VTE & All Patients & $P$ Value \\
\hline \multicolumn{5}{|l|}{ Mechanism of injury } \\
\hline Blunt & $16(55.2)$ & $76(63.9)$ & $92(62.2)$ & .387 \\
\hline Penetrating & $13(44.8)$ & $43(36.1)$ & $56(37.8)$ & \\
\hline \multicolumn{5}{|l|}{ SCI level } \\
\hline Cervical & $17(58.6)$ & $65(55.1)$ & $82(55.8)$ & .033 \\
\hline Thoracic & $12(41.4)$ & $32(27.1)$ & $44(29.9)$ & \\
\hline Lumbar & $0(0)$ & $21(17.8)$ & $21(14.3)$ & \\
\hline Surgery within 3 months of SCI & $0(0)$ & $3(2.5)$ & $3(2.0)$ & .999 \\
\hline Presence of mobility impairment & $20(71.4)$ & $57(47.9)$ & $77(52.4)$ & .025 \\
\hline \multicolumn{5}{|l|}{ Degree of mobility impairment } \\
\hline Paraplegia & $15(75)$ & $44(77.2)$ & $59(76.6)$ & .842 \\
\hline Quadriplegia & $5(25)$ & $13(22.8)$ & $18(23.4)$ & \\
\hline
\end{tabular}

Abbreviations: SCI, spinal cord injury; VTE, venous thromboembolism. 
Table 4. Venous thromboembolism prophylaxis choice by venous thromboembolism development after spinal cord injury, $(n(\%))$.

\begin{tabular}{|c|c|c|c|c|}
\hline Prophylaxis Used & VTE & No VTE & Total Patients & $P$ value \\
\hline No DVT prophylaxis & $0(0)$ & $2(1.7)$ & $2(1.4)$ & $<.001$ \\
\hline SCDs alone without chemoprophylaxis & $7(25)$ & $5(4.2)$ & $12(8.2)$ & \\
\hline Subcutaneous heparin & $6(21.4)$ & $3(2.5)$ & $9(6.2)$ & \\
\hline Subcutaneous heparin and SCDs & $0(0)$ & $3(2.5)$ & $3(2.1)$ & \\
\hline IVC filter & $2(7.1)$ & $0(0)$ & $2(1.4)$ & \\
\hline Enoxaparin & $7(25)$ & $54(45.8)$ & $61(41.2)$ & \\
\hline Enoxaparin and SCDs & $3(10.7)$ & $49(41.2)$ & $52(35.6)$ & \\
\hline Enoxaparin, SCDs, and IVC filter & $1(3.6)$ & $0(0)$ & $1(0.7)$ & \\
\hline Clopidogrel & $0(0)$ & $1(0.8)$ & $1(0.7)$ & \\
\hline Rivaroxaban & $1(3.6)$ & $1(0.8)$ & $2(1.4)$ & \\
\hline Rivaroxaban and IVC filter & $1(3.6)$ & $0(0)$ & $1(0.7)$ & \\
\hline
\end{tabular}

Abbreviations: DVT, deep vein thrombosis; IVC, inferior vena cava; SCDs, sequential compression devices; VTE, venous thromboembolism.

remaining patients experienced penetrating trauma. Injury mechanism was not associated with VTE development, and $55.4 \%$ of SCIs were at the cervical level. Lumbar SCI accounted for $14.2 \%$ of cases, while thoracic SCI accounted for the remaining $29.9 \%$ of cases. SCI level was statistically significantly associated with VTE development. Of individuals that developed VTE, $41.4 \%$ had a thoracic level compared with $27.1 \%$ in those who did not $(P<.05)$. No patients who developed VTE had a lumbar SCI level. Furthermore, of those who developed VTE, $71.4 \%$ had mobility impairment compared with $47.9 \%$ of those who did not $(P<$ $.05)$. The degree of mobility impairment (paraplegia versus quadriplegia) did not appear to influence VTE development. American Spinal Injury Association (ASIA) impairment scale was often recorded at outside rehabilitation facilities and, as a result, was not available for analysis.

Table 4 summarizes VTE prophylaxis choice. DVT prophylaxis consisted of unfractionated heparin $(6.2 \%)$, unfractionated heparin with SCDs (2.1\%), SCDs alone (8.2\%), LMWH (41.8\%), and LMWH with SCDs (35.6\%). Other forms of thromboprophylaxis used included inferior vena cava (IVC) filters, Plavix, Xarelto, and Xarelto with an IVC filter. When comparing DVT prophylaxis, there was a statistically significant reduction in VTE prevalence when SCDs were used with LMWH compared with SCDs alone. Three patients who were given both developed VTE compared with 49 patients who did not. In comparison, 7 out of 12 patients who received SCDs alone developed VTE. There was not a statistically significant difference between the use of LMWH with SCDs and the use of LMWH alone. Heparin use with SCDs was not statistically different from heparin use alone. There was a statistically significant difference between the use of LMWH and unfractionated heparin $(P<$
.001). Seven out of 64 individuals on LMWH developed VTE compared with 6 out of 9 patients on unfractionated heparin.

Average household income was inferred for the entire cohort. Average income for this patient list based on average household income in each individual's residential zip code was $\$ 56647.25$, which is $\$ 30315.75$ below the national average. ${ }^{15}$ Table 2 shows no correlation between healthcare coverage or employment status and VTE development.

\section{DISCUSSION}

Patients who suffer acute SCI are at a high risk of VTE within the following year, with the highest risk period being within the first 3 months. ${ }^{8,16-18}$ Recent studies based on databanks and Western populations show a prevalence ranging from $4 \%$ to $23 \%$, with most studies quoting about a $5 \%$ prevalence despite the use of prophylaxis in a majority of cases. $^{5-7}$ To the authors' knowledge, the $19.59 \%$ VTE rate reported in this study is the highest found in a US cohort. Pierfranceschi et $\mathrm{al}^{7}$ and Clements et $\mathrm{al}^{8}$ determined higher VTE rates. However, these studies were performed in Italy and Australia, respectively, where thromboprophylaxis for SCI may differ from that of the United States and this institution.

The higher prevalence found in this cohort is likely multifactorial and attributable to a combination of baseline patient characteristics and social factors, medical comorbidities, and thromboprophylaxis choice. Current or prior malignancy was statistically significantly associated with VTE development. Patients with a history of malignancy are known to be at risk for DVT development secondary to a hypercoagulable state. Jones et $\mathrm{al}^{5}$ found that metastatic cancer was specifically asso- 
ciated with an increased risk. Further disrupted anatomy from cancer treatment, surgical or radiation, may put these patients at risk. It was not surprising to find that a hypercoagulable state was associated with an increased risk of VTE development.

A novel finding of this study is the association between smokeless tobacco use and VTE development after SCI. Smokeless tobacco, which was often chewing tobacco, also provides a mechanism for increased risk of VTE development. Tobacco and nicotine are known to cause blood vessel narrowing. Narrowing of vasculature may present an opportunity for coagulation and thrombus formation. Interestingly, cigarette smoking was not associated with an increased risk of VTE development. One explanation is that patients who use smokeless tobacco also smoke cigarettes and cigarette smoking is underreported. This would suggest that smokeless tobacco use is an indicator of heavy tobacco and nicotine use with a more pronounced effect on the development of VTE. Further studies are required to assess the pathophysiology behind the effect of smokeless tobacco on VTE development.

Patient mobility has long been reported as an important factor in VTE development. ${ }^{3,9}$ As with the development of DVTs in any stationary patient, peripheral vein pooling can result in blood stasis and thrombus development. Immobile patients suffer a more prolonged form of blood pooling as they can no longer perform skeletal muscle pumping of the extremities. In this cohort, patients who had mobility impairment developed VTE $71.4 \%$ of the time compared with $47.9 \%$ of the time in those who did not. Furthermore, a higher neurologic level of injury was associated with VTE development. Thoracic and cervical injury are associated with a higher risk of VTE development. No patients with a lumbar neurologic level developed VTE. Chung et al ${ }^{17}$ similarly found increased risk of VTE development with thoracic or cervical spine injury when compared with lumbar spine injury. However, the degree of mobility impairment, specifically, was not associated with VTE development. This is in contrast to the findings of Pierfranceschi et $\mathrm{al}^{7}$ and Saraf et $\mathrm{al}^{19}$, which cite paraplegia and quadriplegia, respectively, as risk factors. It is possible that lower degrees of mobility impairment without significant paralysis contributes to the pathophysiology of VTE after SCI. This study was unable to assess ASIA impairment scale classification, as many patients had scoring done at an outside rehabilitation facility where records could not be readily accessed.

The Consortium for Spinal Cord Medicine also suggests that age is associated with an increased risk for development of VTE in their 2016 article on the prevention of VTEs after SCI. ${ }^{9}$ While increased age was suggested by the Consortium for Spinal Cord Medicine to be a risk factor, this study showed no statistical difference between those who did and those who did not develop VTE. One possibility for this is the decreased age at which patients presented. VTE is known to increase in prevalence with age, but many of the patients included in this study were young adults. Godat et $\mathrm{al}^{16}$ found no association between VTE development and age, suggesting that increased age may not be a risk factor for VTE development after SCI.

Chung et $a{ }^{17}$ found a higher VTE rate in patients with hyperlipidemia, diabetes, cerebrovascular attack, atrial fibrillation, or associated lower extremity fracture. This cohort did not demonstrate any association between specific diagnosed medical comorbidities and VTE besides malignancy. It is likely that this patient population has a higher rate of undiagnosed medical comorbidities that contribute to increased VTE prevalence. The surrounding population cared for by this institution has a high prevalence of traumatic injury, gun violence, substance use, and poor medical outcomes. The patients included in this study come from a heavily disenfranchised population. The communities in which study participants live have a mean household income that is approximately $\$ 30000$ below the national average. ${ }^{15}$ Furthermore, the majority of patients in this sample use government-assisted healthcare coverage and are unemployed. No statistical association was found between healthcare coverage or employment status and VTE development. It is the authors' opinion that this relates to the high prevalence of government assistance and unemployment causing a weakness with the power of the study in the control groups.

Patients in this population also have a higher rate of obesity compared with the national average. In this cohort, weight was associated with VTE development, and BMI strongly trended toward significance. Patients who developed VTE on average had a weight that was nearly $8 \mathrm{~kg}$ heavier than those who did not. It is likely that patients with a higher BMI are more likely to have undiagnosed 
medical comorbidities that place them at higher risk of VTE. The population studied in this analysis also has many factors preventing them from improving health, such as food availability and access to care. The findings of this study suggest a greatly increased prevalence of VTE when compared with previous findings in studies conducted in the United States despite the largely appropriate use of anticoagulation. The findings of this study support the suggestion of Jones et $\mathrm{al}^{5}$ that VTE development correlates to factors related to social determinants of health.

This study also showed no statistical difference between any racial or ethnic groups included, so it is unlikely that genetic causes played a large role in increased prevalence. The role of protein $\mathrm{C}$ deficiency and other genetic hypercoagulable states was minimal as no patients carried diagnoses of the aforementioned thrombophilia conditions.

The Consortium for Spinal Cord Medicine's 2016 VTE study suggests the use of LMWH as a first-line anticoagulant with high-dose unfractionated heparin as an alternative in patients who cannot receive LMWH. ${ }^{9}$ Chung et $\mathrm{al}^{17}$ found a VTE rate of $43 \%$ when patients only received mechanical thromboprophylaxis. The findings of this study agree with these suggestions. LMWH was found to effectively lower VTE rates and was found to be the most effective form of chemoprophylaxis in this high-risk population. The current protocol for SCI patients at our institution is to initiate prophylaxis with LMWH 48 hours postoperatively or 48 hours after presentation if patients are treated nonoperatively. This is dosed as either $40 \mathrm{mg}$ daily or $30 \mathrm{mg}$ twice a day depending on physician preference.

A small number of patients were treated with IVC filters in this dataset. Due to the lack of power, independent statistical analysis of IVC filter use compared with LMWH was unable to be performed. Gorman et $\mathrm{al}^{20}$ showed that $20.4 \%$ of patients with SCI treated with IVC filter developed DVT compared with $5.2 \%$ of patients treated without an IVC filter. Their study specifically focused on the long-term development of DVT in patients suffering acute SCI with the use of nonremovable IVC filters. By contrast, Roberts et $\mathrm{al}^{21}$ determined a lower PE rate with IVC filter use in a cervical SCI population. However, the dataset was small, and many patients who may have gone on to develop VTE were lost to follow-up. The authors of this study are hesitant to routinely use IVC filters in patients with SCI unless there are contraindications to chemoprophylaxis. Patients at our institution are often lost to follow-up after hospital discharge, placing them at tremendous risk of complications from retained IVC filters. As secondary or tertiary therapy in patients unable to tolerate chemoprophylaxis, the use of reversible IVC filters with close follow-up would likely be beneficial. However, further studies are required to assess the definitive use of these devices in acute SCI patients.

Limitations of this study include its retrospective nature, which inherently leads to missing datapoints and selection bias. Furthermore, many patients were transferred to a rehabilitation center after several weeks in the hospital, well within the 3-month highrisk time period suggested by the Consortium of Spinal Cord Medicine. Without prolonged monitoring, many of these patients were recorded as having no VTE development in available records. Some patients returned from the rehabilitation facility due to VTE development, but patients who went to other outside facilities and subsequently developed a VTE did not have accessible charts. As a result, the prevalence of VTE in this community is likely higher than our findings suggest. Furthermore, a known complication of SCI is loss of sensation in the extremities. Patients who do not experience pain in the lower extremities are difficult to assess for DVT unless it progresses into a PE. Many patients in this study did not undergo imaging for DVT or did so only following symptom appearance. These patients were recorded as not having developed a VTE.

\section{CONCLUSION}

VTE remains a common complication of patients who suffer acute SCI. This study's inner city urban population with negative social health determinants has a much higher rate of VTE following acute SCI than recent large-scale studies would suggest. The results of this review indicate that nearly 1 in 5 patients from this demographic go on to develop VTE following SCI. In patients without contraindications, LMWH initiated 48 hours postoperatively or after presentation in patients treated nonoperatively appears to be most effective at reducing VTE. Nevertheless, a high index of suspicion for VTE after SCI is required for all individuals in the demographic represented by this cohort, but especially in individuals with a history of malignancy, obesity, tobacco or nicotine use, and higher levels of 
mobility impairment. Standardized venous doppler screening in patients suffering acute SCI may reduce morbidity and mortality by identifying early VTE development before clinical manifestation. Further prospective studies are required to determine the efficacy of this approach along with the optimal timing, dosing, and duration of LMWH prophylaxis. It is also possible that individuals from our demographic would benefit from a longer duration of chemoprophylaxis.

\section{REFERENCES}

1. Geerts WH, Code KI, Jay RM, Chen E, Szalai JP. A prospective study of venous thromboembolism after major trauma. N Engl J Med. 1994;331(24):1601-1606.

2. Toker S, Hak DJ, Morgan SJ. Deep vein thrombosis prophylaxis in trauma patients. Thrombosis. 2011;2011:505373. doi: $10.1155 / 2011 / 505373$

3. Furlan JC, Fehlings MG. Cardiovascular complications after acute spinal cord injury: pathophysiology, diagnosis, and management. Neurosurg Focus. 2008;25(5):E13.

4. Aito S, Pieri A, D’Andrea M, Marcelli F, Cominelli E. Primary prevention of deep venous thrombosis and pulmonary embolism in acute spinal cord injured patients. Spinal Cord. 2002;40(6):300-303.

5. Jones T, Ugalde V, Franks P, Zhou H, White RH. Venous thromboembolism after spinal cord injury: incidence, time course, and associated risk factors in 16,240 adults and children. Arch Phys Med Rehabil. 2005;86(12):2240-2247.

6. Maung AA, Schuster KM, Kaplan LJ, Maerz LL, Davis KA. Risk of venous thromboembolism after spinal cord injury: not all levels are the same. J Trauma. 2011;71(5):1241-1245.

7. Giorgi Pierfranceschi M, Donadini MP, Dentali F, et al. The short- and long-term risk of venous thromboembolism in patients with acute spinal cord injury: a prospective cohort study. Thromb Haemost. 2013;109(1):34-38.

8. Clements R, Churilov L, Wahab ALA, Ng LC. Exploratory analysis of factors associated with venous thromboembolism in Victorian acute traumatic spinal cord-injured patients 2010-2013. Spinal Cord. 2017;55(1):74-78.

9. Consortium for Spinal Cord Medicine. Prevention of venous thromboembolism in individuals with spinal cord injury: clinical practice guidelines for health care providers. Top Spinal Cord Inj Rehabil. 2016;22(3):209-240.

10. Green D. Prevention of thromboembolism after spinal cord injury using low-molecular-weight heparin. Ann Intern Med. 1990;113(8):571. doi:10.7326/0003-4819-113-8-571

11. Ploumis A, Ponnappan RK, Maltenfort MG, et al. Thromboprophylaxis in patients with acute spinal injuries: an evidence-based analysis. $J$ Bone Joint Surg Am. 2009;91(11):2568-2576.

12. Grant RA, Quon JL, Abbed KM. Management of acute traumatic spinal cord injury. Curr Treat Options Neurol. 2015;17(2):334.

13. Teasell RW, Hsieh JT, Aubut J-AL, et al. Venous thromboembolism after spinal cord injury. Arch Phys Med Rehabil. 2009;90(2):232-245.
14. Christie S, Thibault-Halman G, Casha S. Acute pharmacological DVT prophylaxis after spinal cord injury. $J$ Neurotrauma. 2011;28(8):1509-1514.

15. United States Census Bureau. Mean income in the past 12 months (in 2014 inflation-adjusted dollars). https://data. census.gov/cedsci/table?q=income\&tid=ACSST1Y2019.S1901. Accessed September 8, 2020.

16. Godat LN, Kobayashi L, Chang DC, Coimbra R. Can we ever stop worrying about venous thromboembolism after trauma? J Trauma Acute Care Surg. 2015;78(3):475-480; discussion $480-481$.

17. Chung W-S, Lin C-L, Chang S-N, Chung H-A, Sung FC, Kao C-H. Increased risk of deep vein thrombosis and pulmonary thromboembolism in patients with spinal cord injury: a nationwide cohort prospective study. Thromb Res. 2014;133(4):579-584.

18. Gaber TA-ZK. Significant reduction of the risk of venous thromboembolism in all long-term immobile patients a few months after the onset of immobility. Med Hypotheses. 2005;64(6):1173-1176.

19. Saraf SK, Rana RJ, Sharma OP. Venous thromboembolism in acute spinal cord injury patients. Indian J Orthop. 2007;41(3):194-197.

20. Gorman PH, Qadri SF, Rao-Patel A. Prophylactic inferior vena cava (IVC) filter placement may increase the relative risk of deep venous thrombosis after acute spinal cord injury. J Trauma. 2009;66(3):707-712. doi:10.1097/TA 0b013e318188beba

21. Roberts A, Young WF. Prophylactic retrievable inferior vena cava filters in spinal cord injured patients. Surg Neurol Int. 2010;1:68. doi:10.4103/2152-7806.72245

Disclosures and COI: Mr Lowery reports that he has a family member who is employed by Merck $\&$ Co, Inc. None of the products observed in this study were products produced by this company. $\mathrm{He}$ receives no significant funding or financial support from this family member. No funding was provided by this company for this project. As such, there is no perceived financial, commercial, or other conflict of interest. All other authors have nothing to disclose.

Corresponding Author: Andrew Lowery, MD, Temple University Hospital Department of Orthopedic Surgery and Sports Medicine, $3401 \mathrm{~N}$ Broad St, Philadelphia, PA 19140. Phone: (215) 7072111; Email: Andrew.Lowery@temple.edu.

Published 1 June 2021

This manuscript is generously published free of charge by ISASS, the International Society for the Advancement of Spine Surgery. Copyright (c) 2021 ISASS. To see more or order reprints or permissions, see http://ijssurgery.com. 